\title{
Unobtrusive Support System for Prevention of Dangerous Health Conditions in Wheelchair Users
}

\author{
Diego E. Arias, ${ }^{1}$ Esteban J. Pino, ${ }^{1}$ Pablo Aqueveque, ${ }^{1}$ and Dorothy W. Curtis ${ }^{2}$ \\ ${ }^{1}$ Universidad de Concepción, 4070409 Concepción, Chile \\ ${ }^{2}$ Massachusetts Institute of Technology, Cambridge, MA 02139, USA
}

Correspondence should be addressed to Esteban J. Pino; epino@ieee.org

Received 5 February 2016; Revised 24 May 2016; Accepted 1 June 2016

Academic Editor: Giancarlo Fortino

Copyright (C) 2016 Diego E. Arias et al. This is an open access article distributed under the Creative Commons Attribution License, which permits unrestricted use, distribution, and reproduction in any medium, provided the original work is properly cited.

This paper presents an assistive device for wheelchair users with severe disability. It consists of several sensors deployed on a wheelchair, able to sense pressure relief habits, activity level, vital signs, and ambient conditions. A pilot study was conducted to evaluate the potential capabilities of the system and the feasibility of implementing alarms. During a period of two weeks, six full-time wheelchair users were monitored. Results show that the system is able to capture pressure relief patterns and tilt-in-space usage, a mechanism required to avoid pressure ulcers. Data analysis shows that none of the volunteers fully complied with the tilting recommendations. It also shows varied activity levels, both within and between patients, indicating their engagement in daily activities. The system is also able to estimate heart and respiration rate during passive activities (e.g., watching TV and/or resting). Also, high temperatures in summer were monitored while patients were outside, to prevent heatstroke events. All the monitored variables are acquired with unobtrusive sensors, producing no discomfort to the patient and providing valuable information for better care. The final recommendation and alarm system is implemented in a single board computer, to be embedded in the wheelchair.

\section{Introduction}

Wheelchairs users are estimated at about $10 \%$ of disabled people worldwide [1]. The main causes of wheelchair usage are injuries and/or medical conditions that affect the central nervous system (CNS). Depending on how the CNS is damaged, the disability varies. Among the most severe cases are patients going through an advanced stage of a neurodegenerative disease such as Multiple Sclerosis (MS) and patients with tetraplegia or paraplegia who have been affected by a severe injury in the spinal cord.

Besides physical impairment, these patients can be affected by a variety of symptoms such as thermoregulatory problems and cardiorespiratory dysfunction. In sum, they cannot perform daily activity without assistance, and they require continuous nursing care and supervision usually provided by families or specialized caregivers. However, in some cases, the supervision fails and the patients can be exposed to dangerous conditions.

First of all, wheelchair users are prone to develop pressure ulcers (PU) because they spend long hours sitting. PU are injuries on the skin produced by prolonged pressure, usually over parts of the body in contact with the wheelchair. Excessive pressure reduces the blood flow producing tissue ischemia and death, resulting in an ulcer. To avoid PU, patients should relieve pressure frequently. To do that, caregivers should be aware of reminding patients when to relieve pressure and assist them if needed. However, high workload in nursing homes can lead caregivers to forget when the patient should relieve pressure. The same can happen when a family member is responsible for the patient's care. Both situations increase the probability of PU. Secondly, another dangerous situation occurs with disabled patients who suffer thermoregulatory problems. Long periods of time exposed to heat without caregiver's monitoring can produce sunburns, dehydration, heatstroke, and even death. There are reports of deaths produced by heat exposure in assisted living facilities as well as in patient's homes due to impaired self-awareness and failure in supervision [2-4]. Lastly, under severe mobility impairment, people can also develop comorbidities related to respiratory and cardiovascular function which should be constantly supervised by caregivers. For example, people 
with MS can suffer respiratory muscle weakness and bulbar dysfunction which produce difficulty in breathing [5]. Also, the worst cases of spinal cord injury (SCI) are unable to breathe due to paralysis of respiratory muscles requiring ventilation support. Furthermore, this population presents autonomic dysfunction increasing the probability of suffering from heart diseases [6].

The examples described show how wheelchair users with severe disability are constantly at risk. Problems such as low mobility can produce PU, heat sensitivity can produce exacerbation of symptoms and/or heat stress during outdoor activities, and abnormal cardiorespiratory function can affect their health status. In those cases, supervision is very important to prevent dangerous conditions. To this end, a system to monitor variables related to these problems can help caregivers and medical staff to increase patient's quality of life.

\section{Related Work}

Advances in sensors, wireless communication systems, and information technologies make it possible to develop new assistive devices to monitor patients during daily life. Regarding wheelchair bound patients, several research groups have developed monitoring systems to alert patients and caregivers to dangerous situations like those previously described. For example, some groups have focused on developing systems to prevent PU. To this end, authors have deployed several sensors between the patient and the pressure relief cushion, to capture pressure changes. To acquire this information, they use commercial pressure mapping devices [7] and custom sensor arrays based on different technologies such as rigid copper electrodes [8], piezoresistive sensors [9], and force sensitive resistors (FSR) $[10,11]$. Then, data are processed to generate alarms to warn patients and/or caregivers when a change in position is necessary. If any of the sensors detects a dangerous level of pressure during a long period of time, an alarm is generated. Another approach has been used to avoid PU when the patients use powered wheelchairs equipped with a tilt-in-space system. The tilt-in-space system allows modifying the seat angle orientation in relation to the ground while maintaining the seat to back angle [12]. This mechanism allows patients to redistribute the seat pressure, transferring it from the seat to the back. Authors have monitored how often wheelchair users perform a tilt to relieve pressure [13-17]. To this end, the proposed systems are composed of accelerometers able to detect when patients perform a tilt. Based on this information, it is possible to implement alarms to remind patients and caregivers when patients should tilt their chair.

Vital signs such as heart rate (HR) and respiratory rate (RR) are also monitored in wheelchair users. The main difference between the proposed systems lies in the types of sensors used, some of them being more obtrusive than others. For instance, some projects have used conventional sensors such as skin electrodes for ECG and respiration belts which can be difficult to wear, producing discomfort to impaired patients [18]. On the other hand, authors have measured Ballistocardiogram (BCG), defined as the mechanical activity of the heart which is an unobtrusive way to acquire cardiorespiratory activity [19]. To acquire BCG signals, an electromechanical film (EMFi) is deployed on the wheelchair seat and backrest. Using advanced signal processing, it is possible to acquire respiration and heart activity to calculate HR and RR to assess patient's health.

Regarding heat exposure, although there is no report of applications designed for wheelchair users, it is possible to find devices developed to provide an alert to dangerous heat exposure in situations such as workers [20, 21] and car drivers [22] exposed to hot environments. The implemented systems measure ambient temperature $\left(T_{\mathrm{AMB}}\right)$ and relative humidity $(\mathrm{RH})$ to calculate indexes able to estimate thermal discomfort such as heat index (HI) and Wet Bulb Globe Temperature. Also, $\mathrm{HR}$ and $\mathrm{RR}$ are measured to obtain a more reliable estimation of the subject thermal state. Based on this information, alarms are implemented to decide whether people should stop working or whether car drivers are in good conditions to keep driving.

We propose that combining monitoring pressure relief patterns, physiological data, and thermal indexes provides sufficient and timely information about the patient's health while using the wheelchair. To this end, several noninvasive sensors are deployed on a patient's wheelchair to capture vital signs, ambient conditions, pressure relief habits, and patient activity providing useful context-aware information for patients, caregivers, and clinical staff without producing discomfort. Our previous work [23-27] presented different stages of the project, from design to preliminary findings. In this paper, we present the final setup, detailed algorithms, and the use of our assistive device to monitor these important variables during daily life. We also present the final hardware implemented to generate alarms and recommendations to caregivers and to the user. The system is designed to improve the care of wheelchair users who suffer severe mobility impairment by monitoring key parameters known to be relevant during daily activities.

\section{Methods}

3.1. Instrumentation. The system consists of several unobtrusive sensors deployed on a powered wheelchair, able to sense pressure relief habits, patient activity, vital signs, and ambient conditions. The selected sensors are able to capture seat and back pressure changes, tilt angle produced by the tilt-in-space system, respiration and heart activity from BCG signals, $T_{\mathrm{AMB}}$, and $\mathrm{RH}$. To capture pressure relieving habits, nine small FSR (model FSR 406 manufactured by Interlink Electronics) are deployed on the wheelchair to detect prolonged pressure over the buttock and back area. The sensors are distributed as follows: 4 sensors are put on the seat $\left(\mathrm{FSR}_{\mathrm{S}}\right)$, under the pressure relief cushion, and 5 on the backrest $\left(\mathrm{FSR}_{\mathrm{B}}\right)$, inside a piece of foam. To detect the wheelchair tilt and to capture information related to subject activity, an accelerometer (ADXL335 chip) is fixed to the wheelchair. The ADXL335 has analog output and resolution of $\pm 3 \mathrm{~g}$. Based on pressure and accelerometer data, it is possible to detect pressure redistribution produced by tilt-in-space system. To acquire heart and respiratory activity, BCG was measured using EMFi sensors (model L-3030 manufactured by EMFIT Ltd.), a highly sensitive electromechanical film 


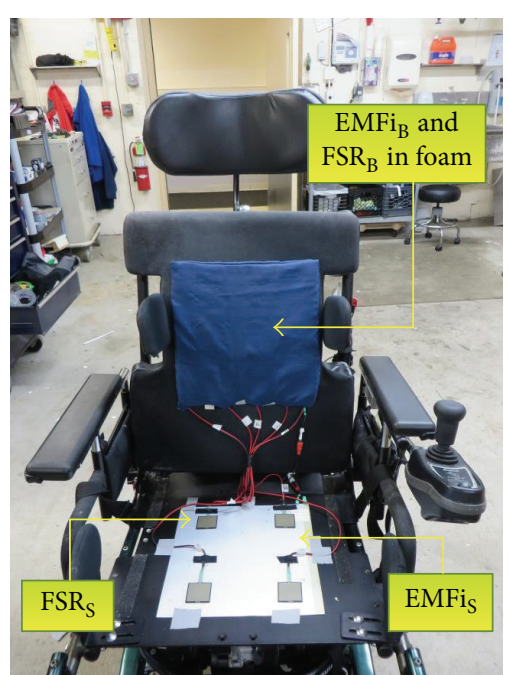

(a)

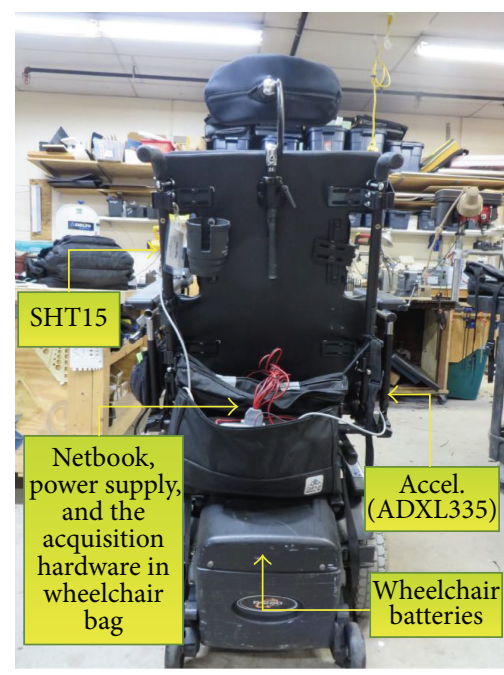

(b)

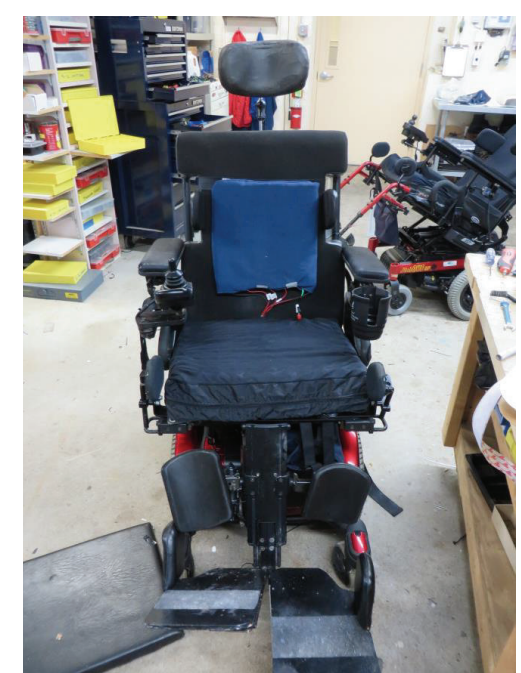

(c)

FIGURE 1: Pictures of the implemented system: (a) front, (b) back, and (c) system ready to use. Unobtrusive sensors are installed on the wheelchair, under backrest foam and pressure relief cushion on the seat. Ambient sensor and accelerometer are attached to wheelchair structure, away from the user. The system is powered by the wheelchair batteries.

sensor able to measure small pressure variations. One sensor was placed on the seat $\left(\mathrm{EMFi}_{\mathrm{S}}\right)$, under the pressure relief cushion, and another was placed on the backrest $\left(\mathrm{EMFi}_{\mathrm{B}}\right)$, inside a piece of foam. Also, an ambient sensor (SHT15 chip manufactured by Sensirion) allows measuring $T_{\mathrm{AMB}}$ and $\mathrm{RH}$ to avoid heat exposure during outdoor activities. This sensor integrates both variables in one chip. Its digital output data are sent to a microcontroller unit (MCU) using a proprietary communication protocol. This setup allows us to capture the information in a noninvasive way without discomfort to the user, because none of the sensors touch the patient directly. All information acquired by the sensors is sent to MCU which samples the data at $100 \mathrm{~Hz}$ with 11 bits of resolution. Then, the data are transmitted through the serial port to a small laptop mounted on the wheelchair. Finally, the data are stored in a PostgreSQL [28] database for further analysis. The whole system is powered by the wheelchair batteries. The power consumed by the system is minimal and does not affect the wheelchair performance. Figure 1 shows the implemented system mounted on the wheelchair.

3.2. PRT Detection Algorithm. An algorithm to detect pressure relief by tilting (PRT) was implemented. PRT is a wheelchair tilt due to the operation of the tilt-in-space system which produces relief of pressure over the buttocks area. This is achieved if the skin is unloaded for at least $3 \mathrm{~min}$ and $30 \mathrm{sec}$ according to the study presented in [29], so the tissues are oxygenated. To ensure this phenomenon, a threshold of $5 \mathrm{~min}$ was used.

The algorithm uses the accelerometer data and the FSR sensors deployed on the seat. First of all, accelerometer data is filtered using a low-pass filter to extract the static component of acceleration and transform it into a tilt angle. The pressure sensors are also filtered to remove high frequency noise due to wheelchair vibration. The angle is averaged using a $10 \mathrm{sec}$ sliding window to detect angle changes. When the tilt angle is reduced by at least 5 degrees, which means that the wheelchair is tilted back, and the pressure sensors reduce their level sharply, the tilt is marked as possible PRT. To consider the tilt as PRT, the subject should maintain this position or increase the tilt angle for at least 5 minutes. Otherwise, the algorithm is restarted. When the subject returns to the initial position, the tilt is finished. Figure 2 shows an example of PRT detection. This full record shows 4 PRT detection instances. Vertical lines marked with " $\mathrm{X}$ " indicate the start of a tilt. Lines marked with "O" indicate the end of a tilt. It is possible to observe how pressure over the buttocks area is transferred to the back during PRT. Also, analyzing the pressure sensors, it is possible to calculate the wheelchair occupancy time. In the example in Figure 2, the volunteer is on the wheelchair for $6: 41$ hours.

3.3. Activity Level Estimation. For wheelchair users with severe disability, a good indication of activity is when they are driving the wheelchair. Therefore, to estimate patient's activity, the dynamic component of the accelerometer was used because it reflects the subject's movement and the wheelchair vibration during driving. To extract this component, the standard deviation of the accelerometer is calculated using a sliding window. High standard deviation values reflect periods of time where the subject shows a high level of activity. Based on tests performed on lab settings, a fixed threshold of 0.5 was used to distinguish between high and low levels of activity as can be observed in Figure 3. Periods of time classified as high activity are used to calculate an activity index (AI) defined as the percentage of the wheelchair occupancy where patients show high activity.

3.4. Ballistocardiogram Processing. Raw EMFi signals are processed to extract respiration and BCG signals. To this end, a filter bank is implemented based on the Discrete Wavelet 


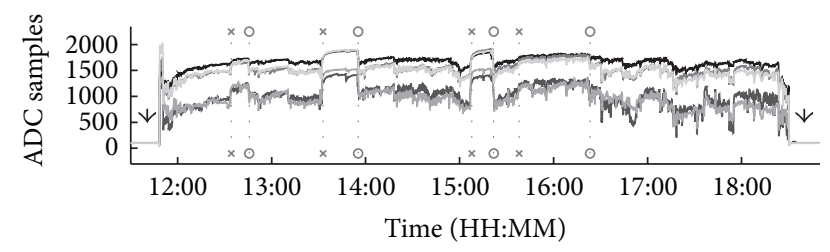

(a)

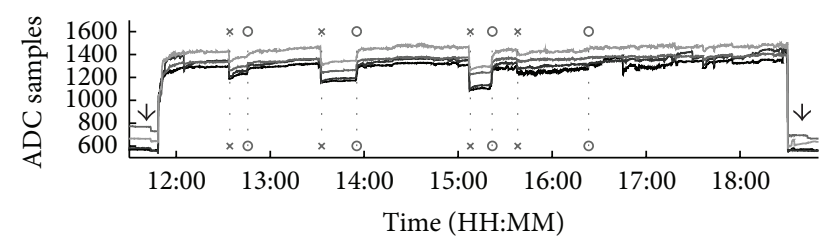

(b)

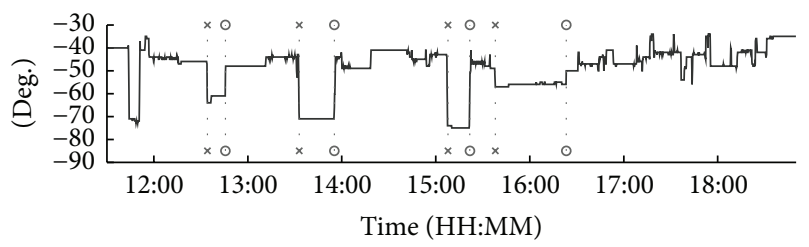

(c)

FIGURE 2: PRT detection algorithm. FSR $_{\mathrm{B}}(\mathrm{a}), \mathrm{FSR}_{\mathrm{S}}$ (b), and tilt angle obtained with the accelerometer (c) allow capturing daily pressure relieving patterns. Vertical lines marked with " $\mathrm{X}$ " and "O" indicate the PRT detected. Arrows on the FSR sensors mark the beginning and end of the wheelchair occupancy.

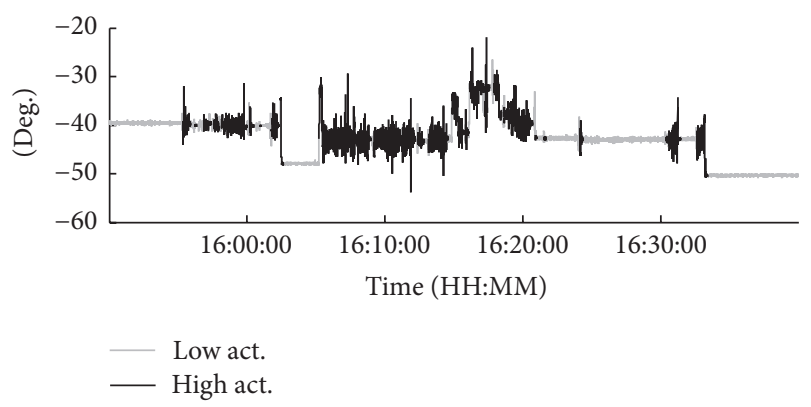

(a)

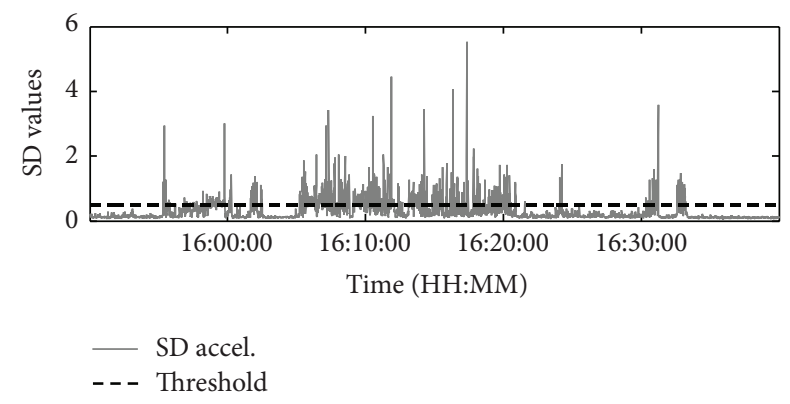

(b)

FIgURE 3: Processing the accelerometer data (a) allows estimating the activity level of each subject. Segments in bold show when the subject presents high activity (e.g., driving, tilting, and moving) as opposed to segments with low activity (e.g., resting, wheelchair stopped). The activity detection is based on the standard deviation of the accelerometer data (b) and a fixed threshold.

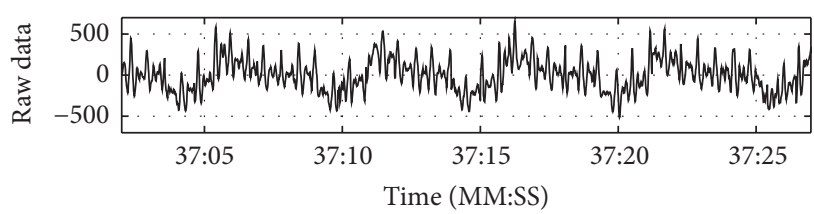

(a)

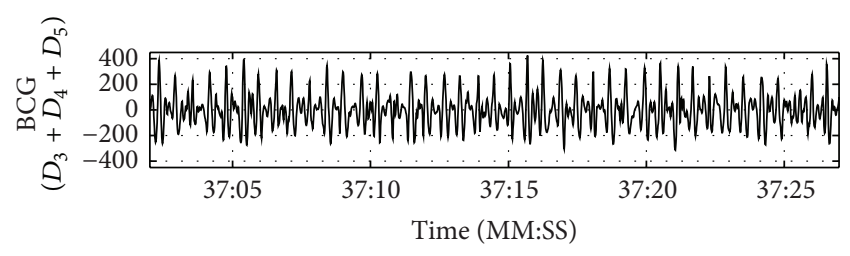

(b)

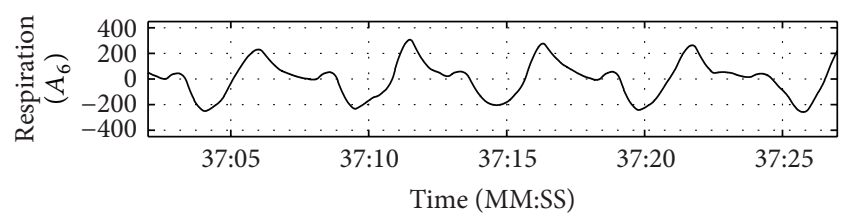

(c)

FIGURE 4: (a) Raw EMFi, (b) BCG, and (c) respiration signals used to obtain vital signs.

Transform (DWT). The DWT filter bank allows decomposing the original signal into $n$ subsignals called details $\left(D_{i}\right)$ and approximations $\left(A_{i}\right)$, where $i$ is the $i$ th decomposition level. The decomposition is done passing the raw data through several low-pass and high-pass filters designed with a specific mother wavelet, selected based on the similarity to the clean BCG waveforms. High frequency information $\left(D_{i}\right)$ and low frequency information $\left(A_{i}\right)$ are contained on the resulting signals; however, to obtain heart and respiratory activity, specific details and approximations must be selected. Raw EMFi data are decomposed into 6 levels using, as mother wavelets, symlet 5 for $\mathrm{EMFi}_{\mathrm{B}}$ and daubechies 10 for $\mathrm{EMFi}_{S}$. An example of BCG and respiratory reconstruction is shown in Figure 4. Respiration signals are reconstructed using $A_{6}$ because it contains frequency components below $0.5 \mathrm{~Hz}$ where respiration activity is found. On the other hand, BCG signals are reconstructed adding the details $D_{3}, D_{4}$, and $D_{5}$ because they contain frequency components between 1 and $10 \mathrm{~Hz}$ where most of the power of the BCG signal is found.

3.5. Respiratory Rate Calculation. An algorithm for RR calculation was implemented using the respiratory signal obtained from raw EMFi data. It works by recognizing inspiration and expiration cycles from respiratory waveform and extracting features such as area, width, and maximum and minimum of each cycle to discriminate valid respiration from artifacts produced by movements. The implemented algorithm was based on [30, 31].

Figure 5 shows a typical respiration waveform where it is possible to observe the features used by the algorithm to detect valid respiration. First of all, the respiratory signal or $R_{\mathrm{LOC}}$ is filtered using a 500-sample moving average to obtain 


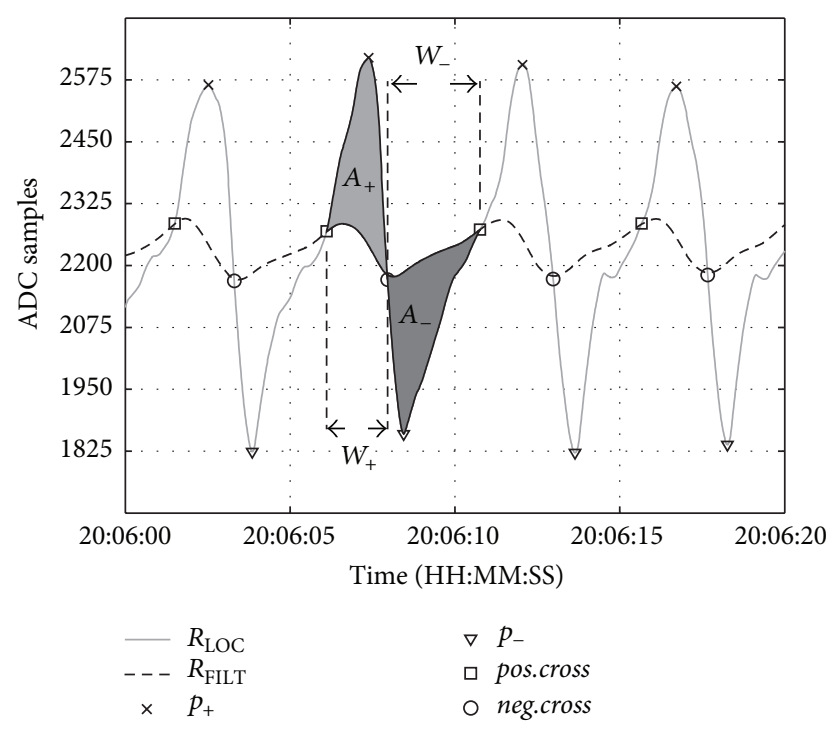

FIGURE 5: Respiration waveform and the features used by the RR algorithm.

its baseline, hereafter called $R_{\mathrm{FILT}}$. It defines 2 cycles according to the crossings with $R_{\mathrm{LOC}}$ : a positive cycle associated with the inspiration, when $R_{\mathrm{LOC}}>R_{\mathrm{FILT}}$, and a negative cycle associated with the expiration, in the other case. These cycles are characterized by their area, width, and maximum and minimum values. The area for the positive cycle $\left(A_{+}\right)$and negative cycle $\left(A_{-}\right)$is defined as $\sum_{i=0}^{N}\left|R_{\mathrm{LOC}}(i)-R_{\mathrm{FILT}}(i)\right|$, where $i$ is the $i$ th sample of $R_{\mathrm{LOC}}$ and $R_{\mathrm{FILT}}$ from the beginning $(i=0)$ to the end $(i=N)$ of the cycle. The width of a positive cycle $\left(W_{+}\right)$is defined as the number of samples from the first crossing (when $R_{\mathrm{LOC}}>R_{\mathrm{FILT}}$ ) to the second crossing (when $\left.R_{\mathrm{LOC}}<R_{\mathrm{FILT}}\right)$. For a negative cycle $\left(W_{-}\right)$, it is defined as the number of samples from the second cross until the third cross (or the first cross for the next respiration). The maximum value of $R_{\mathrm{LOC}}$ during a positive cycle $\left(p_{+}\right)$and the minimum value of $R_{\mathrm{LOC}}$ during a negative cycle $\left(p_{-}\right)$are measured to calculate the amplitude of $R_{\mathrm{LOC}}$ as $\left|p_{+}-p_{-}\right|$.

After a complete cycle, new values of $A_{+}, W_{+}, p_{+}, A_{-}$, $W_{-}$, and $p_{-}$are calculated. Then, the algorithm moves to a decision stage to determine whether valid respiration is detected. The decision stage checks three conditions: (1) the ratio of $W_{+}$to $W_{-}$is greater than 0.3 and less than 1.3 , (2) the ratio of $A_{+}$to $A_{-}$is greater than 0.4 and less than 2.5 , and (3) the absolute amplitude of the current respiratory cycle is greater than $50 \%$ of the amplitude of the previous respiratory cycle.

These thresholds were determined empirically. If all three conditions are met, a valid respiration cycle is detected; otherwise, the cycle is considered noise. To calculate RR, at least two consecutive valid respiration instances must be detected.

3.6. Heart Rate Calculation. An algorithm to calculate HR was also implemented using BCG signals extracted from raw EMFi records. It works by looking for possible beats in zones of BCG signal with high energy.
First of all, the energy of BCG signal is calculated using the following expression:

$$
E_{\mathrm{BCG}}=\sum_{k=i}^{i+q-1}\left|\mathrm{BCG}_{k}\right|^{2}
$$

where $q$ is a $30 \mathrm{~ms}$ sliding window. Then, a peak detector is applied to the energy signal to detect high energy peaks. When a peak of energy is detected, a window of $700 \mathrm{~ms}$ is scanned in the BCG signal to detect potential beats. Consecutives maxima and minima of BCG signal inside the $700 \mathrm{~ms}$ window are used to calculate the Euclidean distance and the slope of the straight line generated between these two points. Once the $700 \mathrm{~ms}$ window is scanned completely, the segment with the longest distance and the minimum slope is considered a potential beat. Finally, if the time between the maximum and the minimum of the selected segment is less than 0.5 seconds, a valid beat is assumed; otherwise, it is treated as noise. Figures 6 and 7 show the flow diagram and an example of the algorithm.

3.7. Heat Index Calculation. $T_{\mathrm{AMB}}$ and $\mathrm{RH}$ measured by the SHT15 sensor were used to calculate the dew point and the HI. These parameters are tabulated in ranges of exposure and the possible effects on human body if the exposure is prolonged. The dew point has been described as a possible indicator of the symptoms exacerbation produced by heat in MS patients [32]. High dew point values (usually over $17^{\circ} \mathrm{C}$ ) can increase the probability of the symptoms worsening. On the other hand, the HI provide warnings due to heat-related illness such as sunstroke, heat exhaustion, and fatigue which can affect a diverse group of at-risk people such as children, the elderly, and people with disabilities. Table 1 shows established ranges of exposure for $\mathrm{HI}$ and dew point.

3.8. Pilot Study. A pilot study was conducted to evaluate the capabilities of the system [25]. To this end, volunteers were recruited from the Boston Home (TBH), a specialized care residence for people with advanced MS and other neurological diseases. To participate in this study, all participants should be full-time wheelchairs users with severe disability. Also, their wheelchairs should be electric-powered and equipped with at least a tilt-in-space system and an inflatable pressure relief cushion as methods to relieve pressure.

The protocol consists of deploying the prototypes on the wheelchairs at the beginning of the study. Every day, before the volunteers start to use their wheelchairs, the prototypes are checked to avoid technical issues. Then, prototypes collect data continuously during the whole time spent on the wheelchair. This study was approved by the MIT Committee on the Use of Humans as Experimental Subjects.

\section{Results}

The study was conducted during a period of 2 weeks. Six TBH residents consented to participate in this study: 4 women and 2 men (hereafter called S1 to S6) with a mean age of $56 \pm 12$ years. All were diagnosed with a progressive form of MS. The 


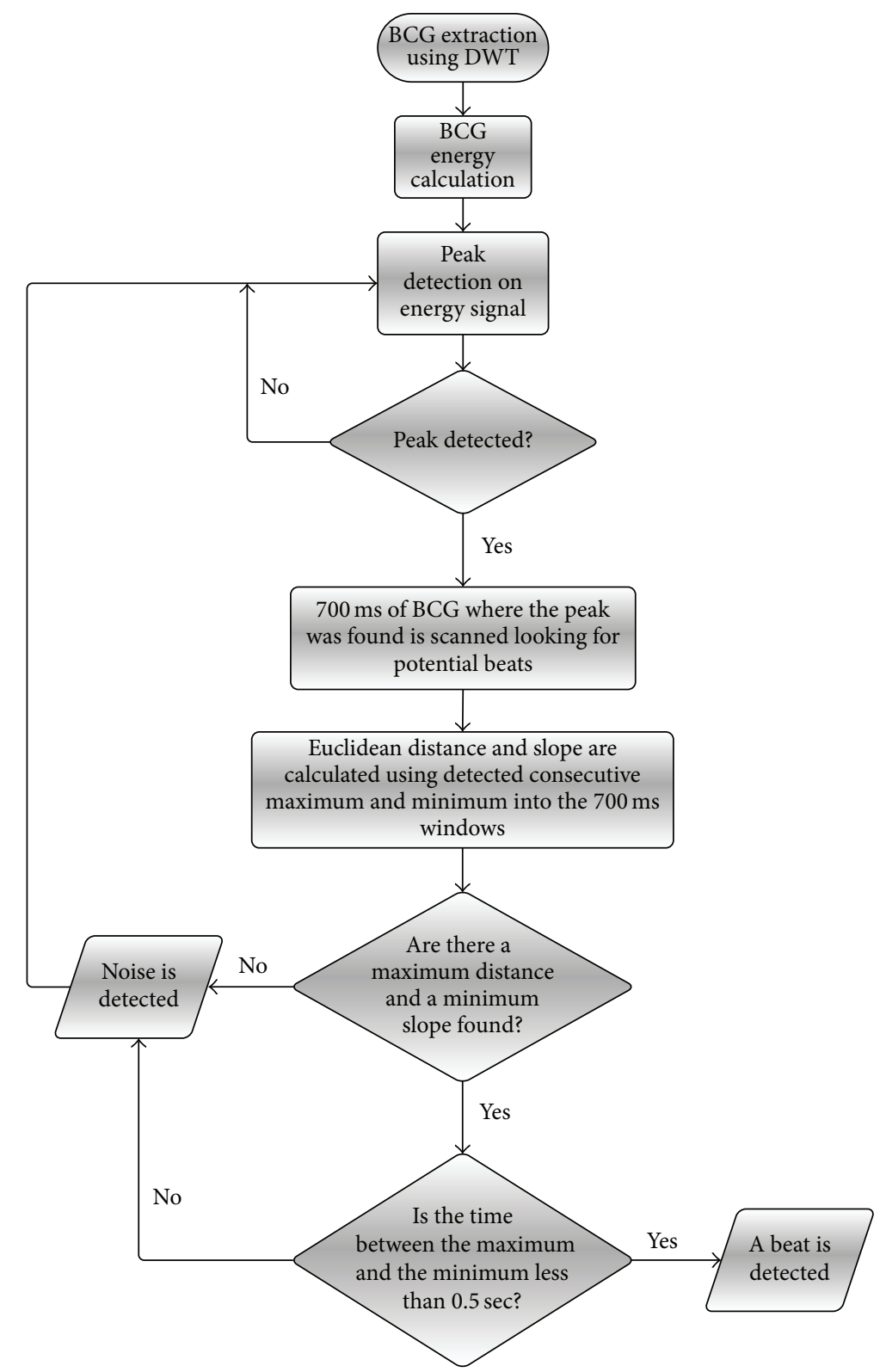

FIGURE 6: Flow diagram of the peak detection algorithm to calculate HR from BCG.

TABLE 1: HI effects on people in high risk group and effects of dew point on MS.

\begin{tabular}{|c|c|c|c|}
\hline \multicolumn{2}{|c|}{ Heat index } & \multicolumn{2}{|c|}{ Dew point } \\
\hline $\mathrm{HI}\left({ }^{\circ} \mathrm{C}\right)$ & General effects & Dew point $\left({ }^{\circ} \mathrm{C}\right)$ & MS symptoms \\
\hline Above 54 & $\begin{array}{l}\text { Highly likely heat or sunstroke with } \\
\text { continued exposure }\end{array}$ & Above 17 & Severe \\
\hline $41-54$ & $\begin{array}{l}\text { Likely sunstroke, heat cramps, or } \\
\text { heat exhaustion; possible heatstroke } \\
\text { with prolonged exposure and/or } \\
\text { physical activity }\end{array}$ & $14-17$ & Moderate \\
\hline $32-40$ & $\begin{array}{l}\text { Possible sunstroke, heat cramps, or } \\
\text { heat exhaustion with prolonged } \\
\text { exposure and/or physical activity }\end{array}$ & $11-15$ & Slight \\
\hline $26-31$ & $\begin{array}{l}\text { Possible fatigue with prolonged } \\
\text { exposure and/or physical activity }\end{array}$ & $5-10$ & Very slight \\
\hline
\end{tabular}




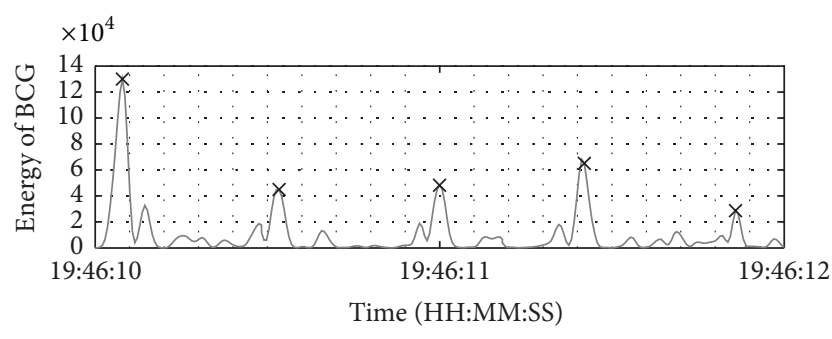

$\times$ Energy peaks

(a)

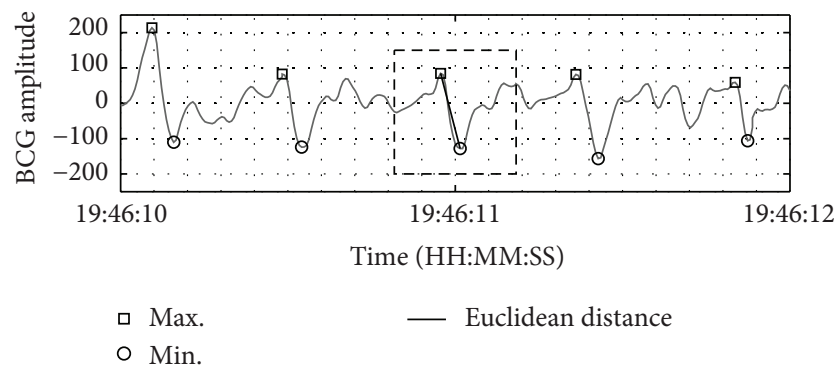

(b)

Figure 7: (a) Peak detection over the energy of BCG. (b) Beat detection over BCG signal. The dashed line rectangle indicates the $700 \mathrm{~ms}$ window.

mean time after diagnosis was $24 \pm 6$ years. The Expanded Disability Status Scale [33] varies between 7.5 and 8.5 which indicates a high disability level. All participants use a pressure relief cushion manufactured by $\mathrm{ROHO}^{\circledR}$.

Figure 8 shows the AI and the wheelchair occupancy distribution from all subjects during the period of 39 days of data collection. On average, the participants presented an activity index of $18.6 \pm 11.3 \%$ with a maximum of $61.1 \%$ and minimum of 5\%. In most cases (29 days), the subjects showed an AI between $10 \%$ and $30 \%$. Only in 2 days $(2.2 \%$ of time) did they present an AI greater than 30\%. Regarding the wheelchair occupancy, the participants spent on their chairs $6.38 \pm 1.72$ hours per day. The maximum occupancy registered was 10.58 hours and the minimum was 2.17 hours. On most days (17 days), the subjects spent between 4 and 6 hours $(44 \%)$ on their wheelchairs. Only in 3 days $(7.7 \%)$ was the wheelchair occupancy less than 4 hours. On the rest of the days, the occupancy time was distributed as follows: $6-8 \mathrm{hrs}$ in 12 days (31\%), 8-10 hrs in 6 days (15.4\%), and over $10 \mathrm{hrs}$ in 1 day (2.6\%). Figure 9 shows a full report of activity and wheelchair occupancy registered for S1. On average, S1 used her chair $7.47 \pm 0.92$ hours/day. During this time, she showed an AI between $20 \%$ and $30 \%$, except for day 3 where the AI reached $48 \%$. The increase in the AI is coincidental to when S1 left the nursing home to go to a shopping mall.

There are several recommendations about the frequency to relieve pressure. Some guidelines recommend relieving pressure every $15 \mathrm{~min}$ [34]; other guidelines recommend longer intervals such as every 1 hour or every 2 hours [35]. Only $2.1 \%$ of PRT performed by the subjects meet the recommendation if the interval is every $15 \mathrm{~min}, 25.8 \%$ if the interval is every 1 hour, and $57.7 \%$ if the interval is every 2 hours. For instance, Figures 10 and 11 show two different cases of pressure relief patterns: a subject who performs several PRT and another who does not. Figure 10 shows a full record from S1 during day 9, where the subject performed 10 PRT during a period of 9.47 hours on the chair. Each tilt lasted $16 \mathrm{~min}$, on average. On the other hand, Figure 11 shows S4 performing only 1 PRT during a period of 10.58 hours spent on the wheelchair. In this case, each tilt lasted 1.25 hours on average. It is also possible to observe that the subject only touches the sensors on the backrest when tilting which can be an indication of bad posture.

Figure 12 shows a histogram that reflects the time intervals spent by the subjects on their chairs without performing a PRT. S1 and S2 achieved shorter intervals. $18.9 \%$ of the intervals for S1 and 5.1\% for S2 were under 1 hour. For S1, most of the intervals (41.7\%) were between 1 and 2 hours. On the other hand, more than $50 \%$ of the intervals spent by the rest of the subjects (S2 to S6) were longer than 3 hours, exceeding all recommendations to avoid $\mathrm{PU}$. The maximum was registered by S6, with $93.9 \%$ of the intervals over 3 hours. There were days when S2 to S6 did not perform PRT at all. For instance, S3 spent 3.57 hours without tilting the chair during day 6 . If this situation is frequent, the subject is prone to develop PU. However, the situation is more risky when the wheelchair occupancy is high. For instance, S6 spent 9.18 hours on the chair without performing a PRT during day 1, four hours more than S3, increasing the probability of developing PU. The average duration in a PRT position is 1.57 hours. However, those who perform frequent PRT spent a shorter time in a tilted position than the subjects who do not. For instance, S1 spent 21 minutes in a tilted position on average. On the other hand, S6 spent around 4.5 hours in this position.

Regarding vital signs calculation, raw EMFi signals were acquired and processed to estimate $\mathrm{HR}$ and RR. As data was collected during patient's daily life, signals were severely affected by noise. Situations like wheelchair driving, operation of the tilt-in-space system, wheelchair crashes, subject's movements, and any technical issues that affect signal quality destroy BCG and respiratory cycles, rendering it impossible to estimate vital signs. However, when the subjects stay calm on their wheelchairs during passive activities (e.g., taking a nap, watching TV, and/or resting), it was possible to estimate vital signs. For example, Figure 13 shows over 1 hour of record obtained from S1. During this period, the HR and RR averages were $90.9 \pm 6.2$ beats/min and $14.7 \pm 0.8$ breaths $/ \mathrm{min}$, respectively. It is possible to observe clean segments of the raw EMFi signal as well as segments that are affected by severe noise (marked with rectangular areas). HR and RR trends were estimated only when clean signals are detected. The algorithms are stopped to reduce false detection in noisy segments. The collected data show that raw EMFi signals are clean between $40 \%$ and $50 \%$ of the wheelchair occupancy time.

In the study, it was usual to observe the residents resting in their chairs or taking a nap. During this time, the system was able to capture respiratory abnormalities such as apneas. Figure 14 shows a $5 \mathrm{~min}$ record captured from S3 where it is possible to observe 4 consecutive apneas lasting more than $15 \mathrm{sec}$ each, a condition that would be very difficult to 


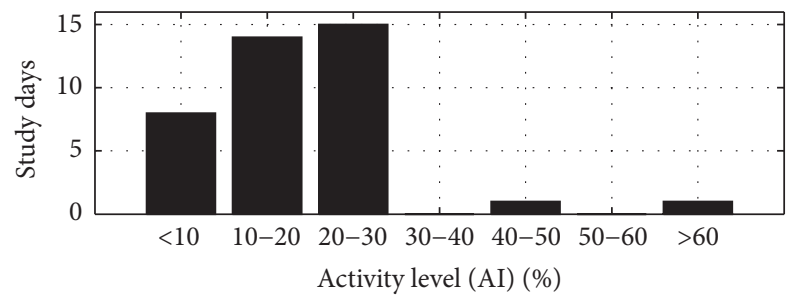

(a)

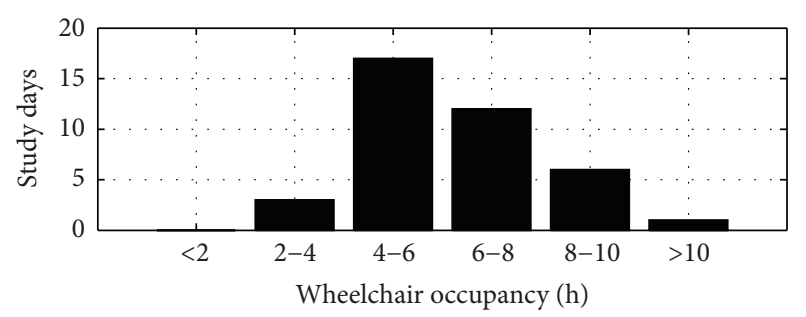

(b)

FIGURE 8: (a) AI and (b) wheelchair occupancy for all subjects during a period of 39 days of data collection.

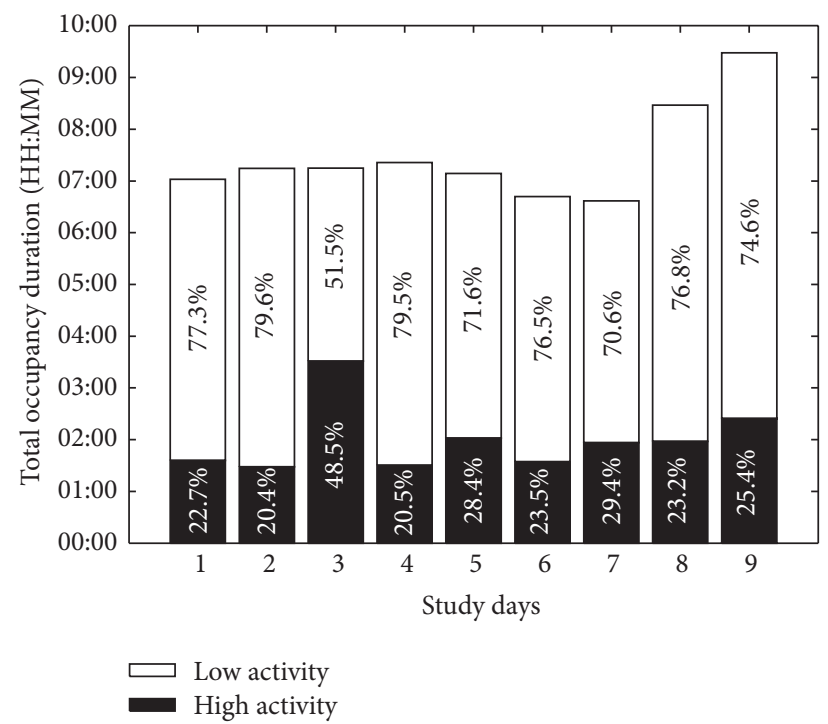

FIGURE 9: Wheelchair occupancy and AI registered by S1 during a period of 9 days of study.

detect otherwise. Apnea events, usually associated with sleep apnea, affect the cardiovascular system producing progressive damage to the heart [36].

As mentioned before, people with severe mobility impairment are very sensitive to heat, which can produce exacerbation of the symptoms and/or heat-related illness. For this reason, caregivers should be extra careful when patients spend time outside in summer. For example, TBH provides some recommendations for residents and caregiver staff to avoid dangerous heat exposure. The recommendations include checking patient's status every 30 min while they are outside and avoiding exposure to $T_{\mathrm{AMB}}$ above $35^{\circ} \mathrm{C}$ or $\mathrm{HI}$ greater than $35^{\circ} \mathrm{C}$. However, the protocol relies on human evaluation and is susceptible to failure. Sometimes high workload in nursing homes can lead to forgetting checking on patients as what happened in [2] with fatal consequences. The implemented system allows monitoring ambient conditions continuously, which can be used for a potential alarm system. For example, Figure 15 shows a full-day record of $T_{\mathrm{AMB}}$ and $\mathrm{RH}$ used to calculate $\mathrm{HI}$ and dew point value. It is of note that the wheelchair user went outdoors for almost an hour, a period of time where the temperature rose to $30^{\circ} \mathrm{C}$. These values are classified both by the $\mathrm{HI}$ and by the dew point as

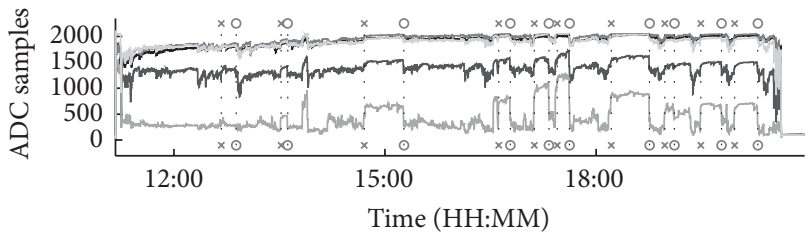

(a)

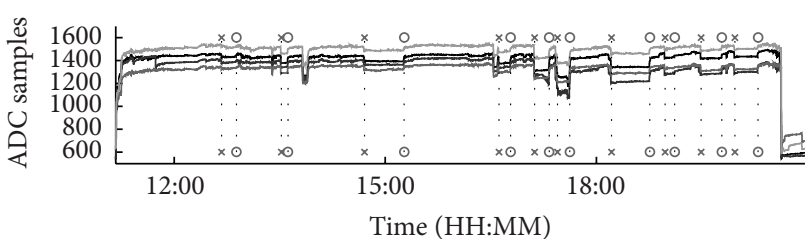

(b)

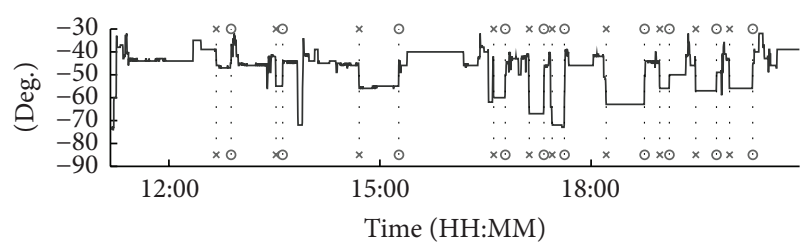

(c)

FIGURE 10: Pressure and angles recorded from S1 wheelchair for 9.47 consecutive hours. (a) FSR $\mathrm{B}$, (b) FSR , and (c) angle data are labeled with the PRT detection. In this case, S1 registered 10 PRT, with an average tilt duration of $16 \mathrm{~min}$.

dangerous exposure, implying that the patient should go back inside.

\section{Final Hardware}

The final system was implemented in a single board computer (SBC) in order to reduce the power consumption and have a self-contained system that does not require external processing. The SBC is a Raspberry Pi 2, Model B running Raspbian Jesse Linux OS. The algorithms and the alarm system were programmed in Python. The main characteristics are shown in Table 2.

5.1. Single Board Computer Setup. The SBC and its main accessories are shown in Figure 16. The MCU acquires data from the 4 sensor types: EMFi, FSR, ADXL335, and SHT15. Then, data is labeled and packaged and sent to the SBC 


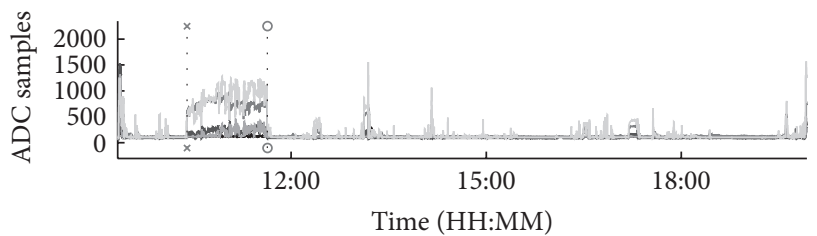

(a)

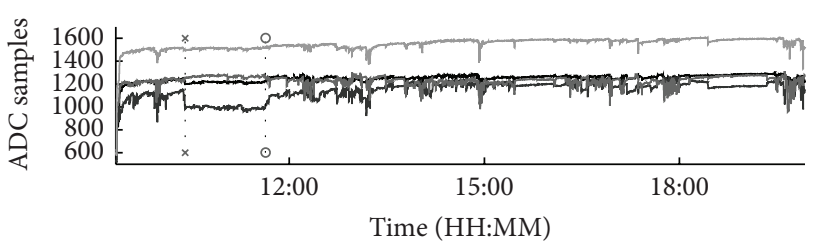

(b)

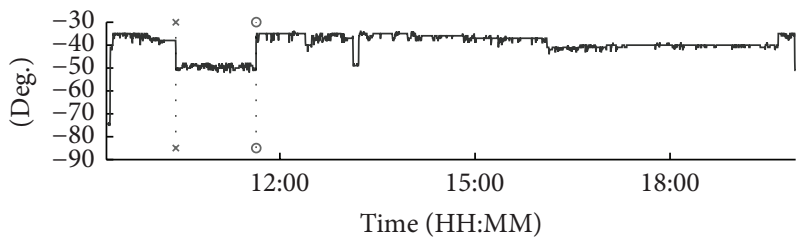

(c)

FIGURE 11: Pressure and angles recorded from S4 wheelchair for 10.58 consecutive hours. (a) $\mathrm{FSR}_{\mathrm{B}}$, (b) FSR , and (c) angle data are labeled with the PRT detection. In this case, S4 presents only one PRT lasting 1.25 hours.

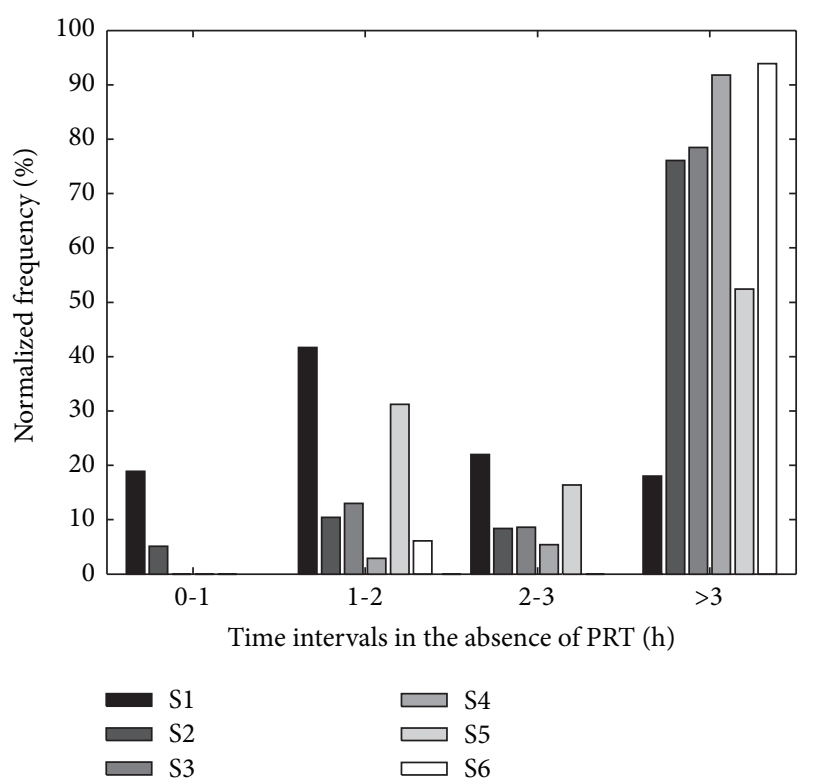

FIGURE 12: Time intervals in the absence of PRT obtained from all subjects.

through RS-232/USB adapter, at $57.6 \mathrm{kbps}$. The SBC runs the tilt, activity, HR, RR, apnea detection, and heat index algorithms to process the information from the sensors and issues alarms or recommendations to the user. The notifications are issued using recorded voice messages sent through a standard $3.5 \mathrm{~mm}$ jack into a powered speaker. When the system is
TABLE 2: Technical specifications for Raspberry Pi 2 SBC.

\begin{tabular}{lc}
\hline Characteristic & Value \\
\hline CPU & $900 \mathrm{MHz}$ quad-core ARM Cortex-A7 \\
RAM & $1 \mathrm{~GB}$ \\
Storage & $8 \mathrm{~GB}$ SDHC class 10 card \\
OS & Raspbian Jessie Linux \\
Audio and video & $3.5 \mathrm{~mm}$ jack, HDMI \\
Size & $85.6 \mathrm{~mm} \times 53.98 \mathrm{~mm}$ \\
Power consumption & $800 \mathrm{~mA}(4.0 \mathrm{~W})$ \\
Power supply & $5 \mathrm{~V}$ through micro USB or GPIO header \\
\hline
\end{tabular}

connected to the Internet via $\mathrm{WiFi}$, an email is also sent to the configured account to alert a caregiver.

5.2. Alerts. The programmed alerts can be divided into 3 groups.

(i) General Information. This group comprises the following:

(i) "The system is on": this indicates the start of the data processing, once a person is detected in the wheelchair

(ii) "The system is off": this indicates the end of data processing and is issued when the pressure sensors indicate that the person has left the wheelchair.

(ii) Heat Index Alarms. This group comprises the following:

(i) "Please, go to a cooler place": this is issued when the user has been in the $26-31^{\circ} \mathrm{C}$ range for more than 1 hour or in the $32-40^{\circ} \mathrm{C}$ range for more than 30 minutes.

(ii) "You must go to a cooler place": this is issued when the user has been in the $41-54^{\circ} \mathrm{C}$ range for more than 20 minutes.

(iii) "You must go to a cooler place, immediately": this is issued when the user has been in the highest heatstroke level, over $54^{\circ} \mathrm{C}$, for more than 5 minutes.

(iii) Tilting. This group comprises the following:

(i) "Please, tilt the chair": a tilt is recommended after one hour without PRT.

5.3. SBC Performance. The performance of the SBC was evaluated in terms of power consumption and processing load. The average power consumption is $0.374 \mathrm{~A}$ at $5 \mathrm{~V}$. This is $56 \%$ lower than the first version using a netbook. The whole system, considering the MCU and sensors, requires $0.624 \mathrm{~A}$. The average load in $60 \mathrm{~s}$ of the SBC is $8.05 \%$. The Python program uses $7 \%$, leaving enough processing power for other tasks.

\section{Discussion}

The implemented system captures pressure relief habits, activity level, vital signs, and ambient conditions unobtrusively during daily activities. Analyzing the data acquired 


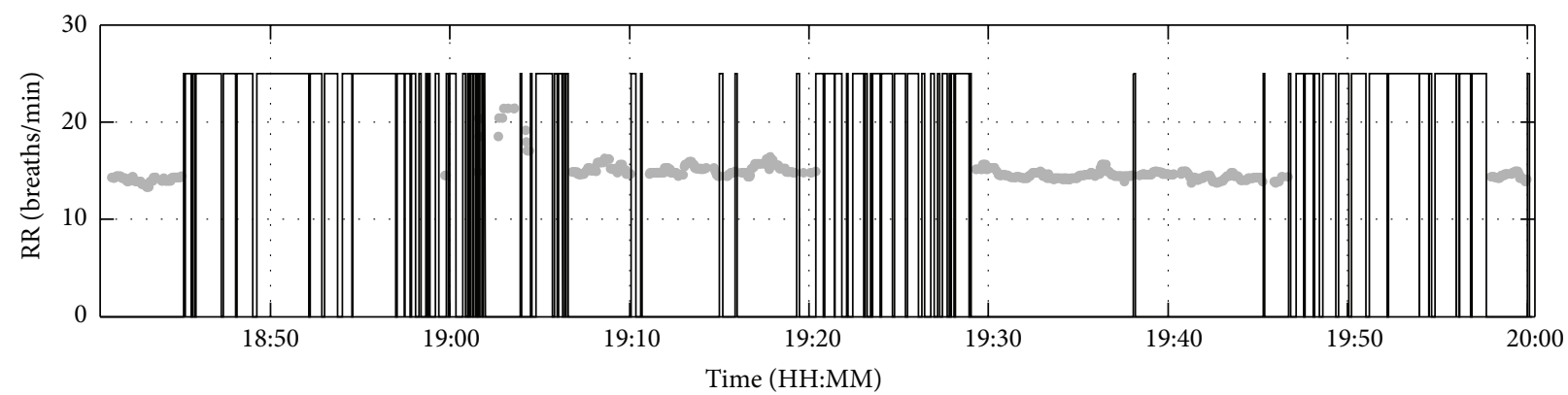

(a)

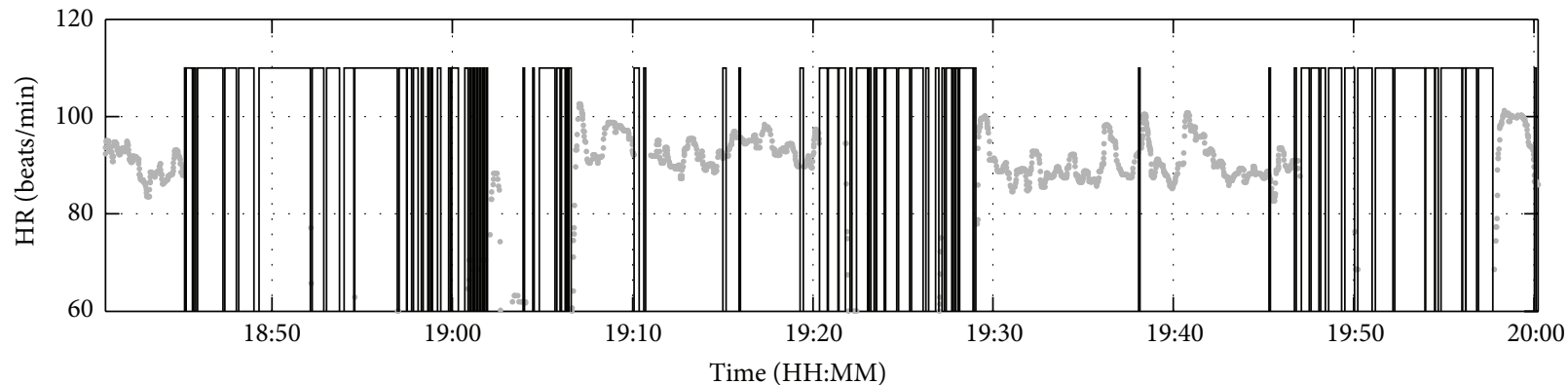

(b)

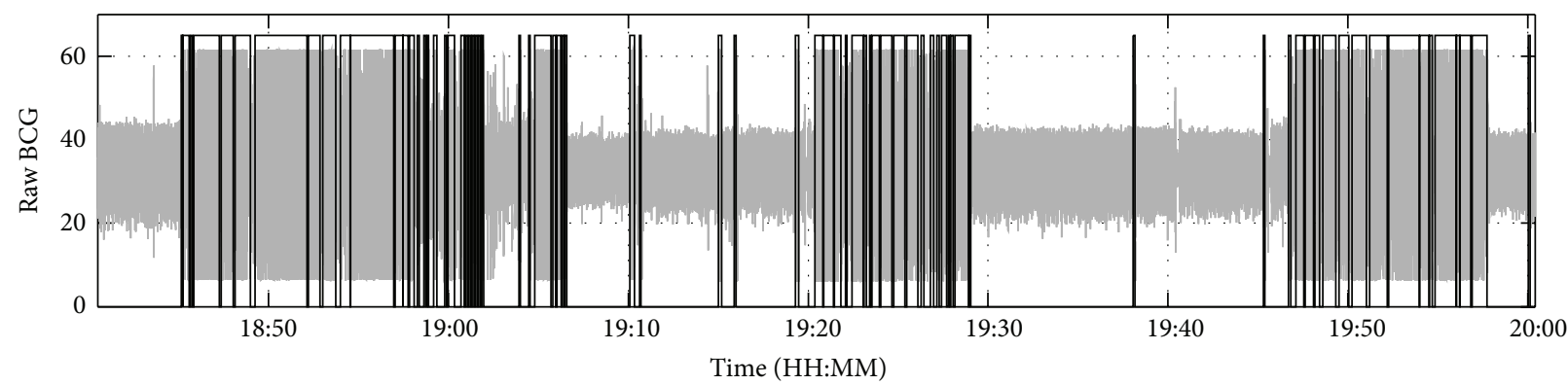

(c)

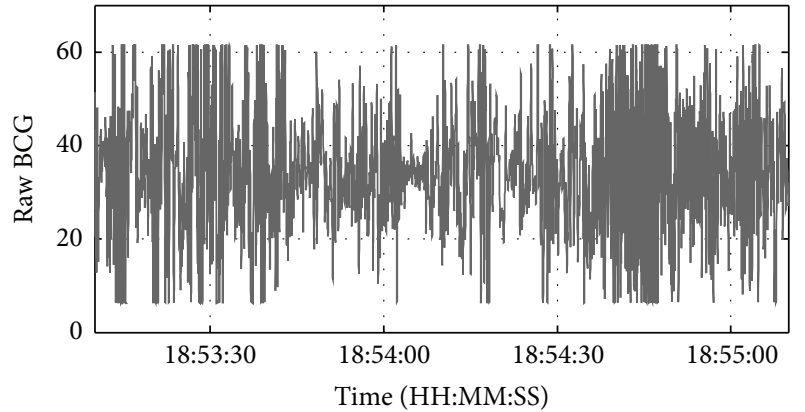

(d)

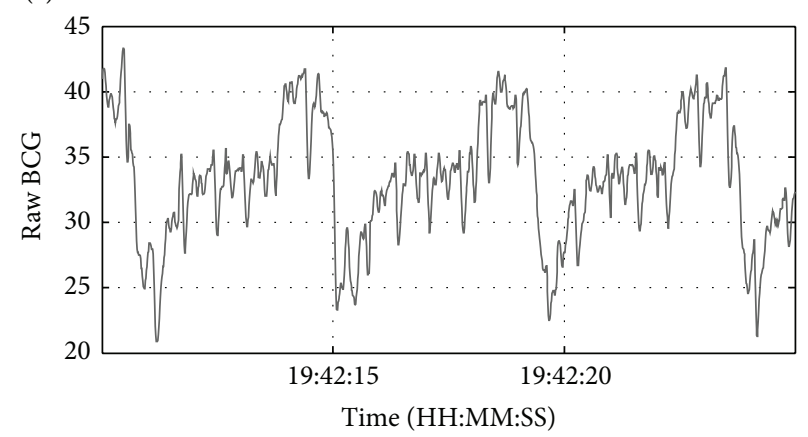

(e)

FIGURE 13: (a) RR and (b) HR series obtained from raw EMFi signals. Rectangular areas mark the zones of (c) BCG signal detected as noise. Vibration during wheelchair driving and subject's movements produces (d) noise signals. When this occurs, the algorithms to calculate HR and RR are stopped. Only during (e) clear BCG signals are vital signs estimated.

from noninvasive sensors, useful parameters are obtained such as wheelchair occupancy, activity level, PRT per day, heart and respiration rate, apneas, and ambient heat index, which are useful to suggest actions that can improve life quality or to provide objective information to clinicians about social engagement and physiological status.

Multiple data sources are combined to determine the status of the subject. During activity periods, as determined by the accelerometer, the HR and RR algorithms are turned off. PRT is validated when both accelerometer and pressure sensors show that pressure relief has been produced. However, advanced methods of data fusion were not included for lack of a better framework to integrate sensor data [37]. For instance, RR is present in both the FSR and the EMFi sensors, but the information is only extracted from EMFi. Also, as proposed in [38], valuable information could be obtained 


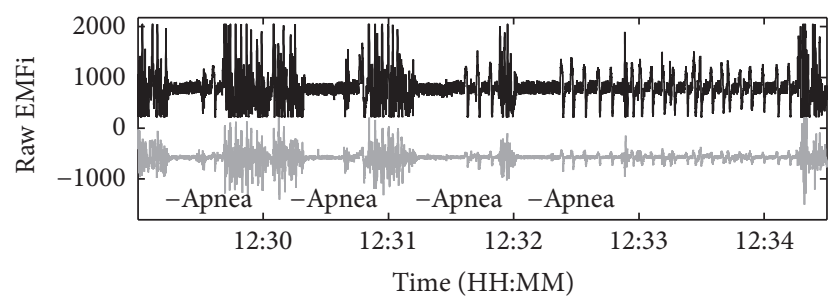

(a)

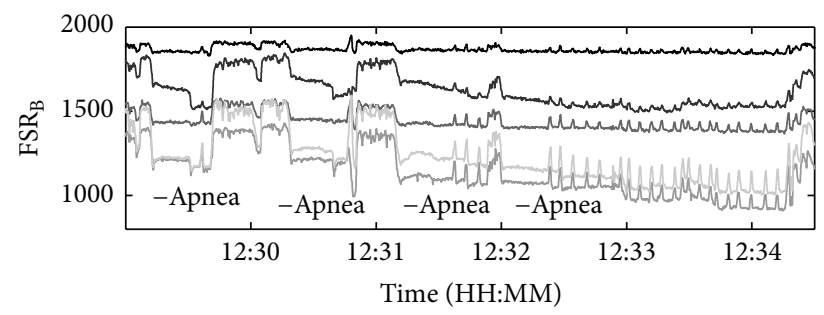

(b)

FIGURE 14: Four consecutive apneas captured by (a) the EMFi and (b) the $\mathrm{FSR}_{\mathrm{B}}$ sensors.

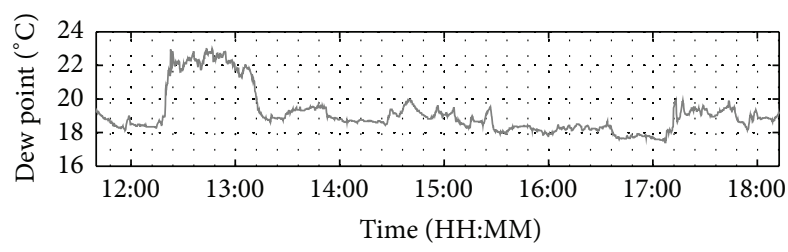

- Dew point

(a)

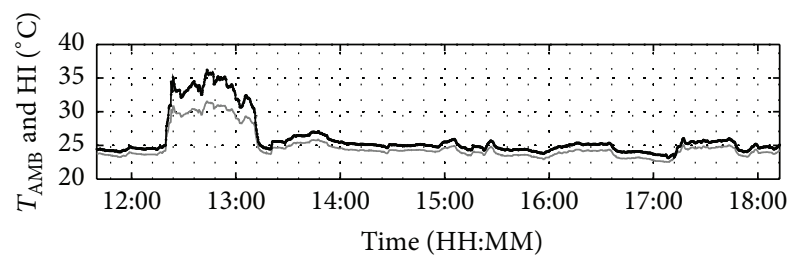

$-T_{\mathrm{AMB}}$

$-\mathrm{HI}$

(b)

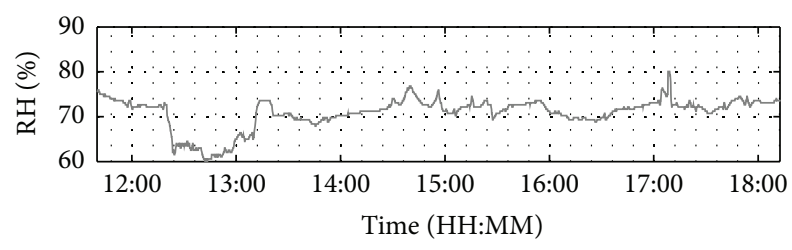

$-\mathrm{RH}$

(c)

Figure 15: HI and dew point data as a wheelchair user moves outdoors after lunch, in the summer. (a) Calculated dew point, (b) $T_{\mathrm{AMB}}$ from the sensor and calculated $\mathrm{HI}$, and (c) RH from the sensor.

from the social interactions between users of the system, as a proxy for mental decline.
The pilot study conducted shows that most of the subjects spent long periods of time on the wheelchair, but they present a low activity level (AI between $10 \%$ and 30\%) due to their severe disability. However, we also found some participants who spent a short time on it (wheelchair occupancy $<4$ hours in $7.7 \%$ of the monitored days). This can be explained by the fact that the volunteers recruited for this study are affected by advanced MS, which is characterized by fatigue and/or depression [39]. These symptoms seriously affect the mood and the capacity to perform daily activities which can lead to spending more time in bed. In this case, the usage of this assistive device could be complemented with nursing care and/or assistive devices focused on the time spent in bed.

The results also show that the subjects did not relieve pressure in a proper way using the tilt-in-space system. This result is similar to those presented in [15]. In most cases, the subjects did not comply with the recommendations related to the frequency of pressure relief. They spent long periods (over 3 hours) without performing a PRT. In addition, during the study, there were entire days when the subjects did not perform PRT. If these situations recur frequently and the subjects spend long hours on the chair without relieving pressure, the probability of PU increases.

No pressure changes and tilt absence during long period of time should cause a warning to be sent to caregivers/family member or patient to avoid pressure ulcers. The designed system will allow implementing an alarm system which helps remind the subjects when they should tilt their chair. The collected data shows the feasibility of implementing just-intime personalized alarms or reminders for PRT with a much better expected outcome than general broadcast reminders or "once in a while" nursing reminders. In this study, the system was deployed for wheelchair users living in a nursing home; however, it can also provide valuable assistance in a homecare environment.

Regarding vital signs, $\mathrm{HR}$ and RR were obtained from raw EMFi signals. Although there are periods of time where it is not possible to estimate vital signs due to severe artifacts, the system is able to acquire information that it is not collected normally. Usually caregivers measure vital signs once a day using methods such as taking the pulse on the wrist or counting the patient's breaths. The obtained vital signs are useful to improve patient's care through assessing their vital signs continuously to quickly assist in case of emergency. RR information was also useful for detecting apnea events during the day. This was a particularly interesting finding, because, up to this point, caregivers could only register apnea events through obtrusive and uncomfortable methods such as polysomnography. Also, measuring vital signs allows knowing a patient's normal ranges considering their impaired condition. During the data collection, it was clear that even a simple "noninvasive" pulse oximeter to capture HR can produce discomfort in patients with advanced chronic diseases such as MS. For this reason, it is important to look for alternative ways of measuring vital signs in these patients.

In relation to heat exposure, which is a real concern in assisted living facilities, the collected data show that patients are exposed to high temperatures which can affect their health status. Reminders targeted at the wheelchair user 


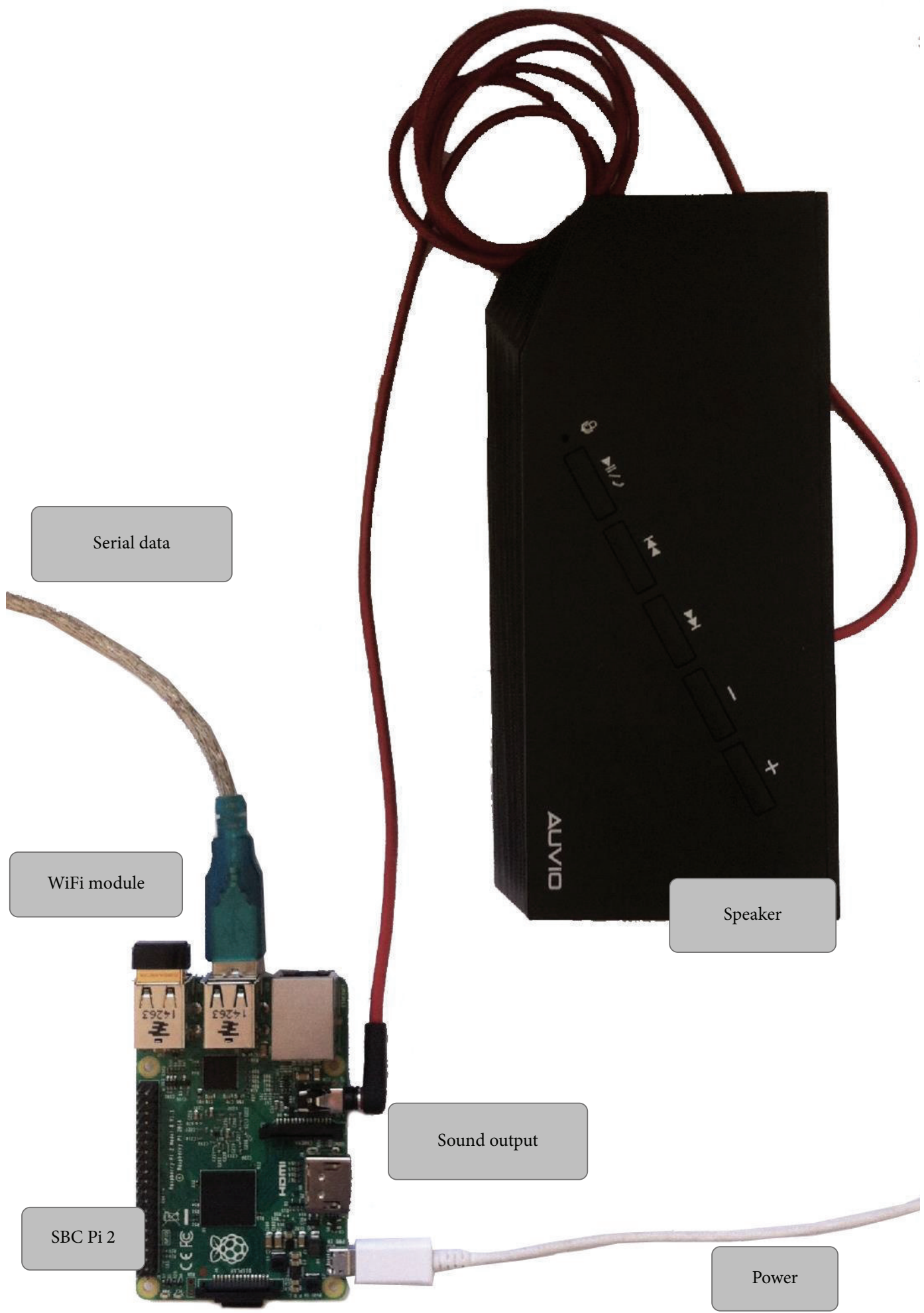

FIGURE 16: Raspberry Pi 2 board with USB serial data connection, WiFi module, external speaker, and micro USB power source.

and/or caregivers regarding current ambient conditions also contribute to preventing risky exposure and improving care.

All these alarms and reminders were successfully programmed on a Raspberry Pi 2 Model B $(900 \mathrm{MHz}$ quadcore ARM Cortex-A7, 1GB RAM, Raspbian Jessie Linux).
This single board computer is powerful enough to replace the netbook used for the original data collection. In the Raspberry Pi 2, a Python program is in control of issuing context-aware recommendations such as "Please, tilt the chair" after a long time in the same position or "You must go 
to a cooler place, immediately" when in risk of heatstroke. For medical conditions, such as abnormal heart rate, respiration rate, and apneas, an email is sent to the appropriate caregivers. The system is performing as expected.

\section{Conclusion}

The proposed system with the selected unobtrusive sensors was able to detect daily activity events in long-term wheelchair users. All sensors were able to provide sufficient ambient and physiological information to provide contextaware recommendations, both to the user and to the caregivers to improve care. The real-time conditions considered to develop a reminder and alarm system from the acquired data are (1) normal condition, (2) pressure relief warning, (3) heatstroke warning, (4) abnormal HR (high or low), and (5) abnormal RR (high or low and apneas).

Conditions (1) and (2) are best communicated directly to the wheelchair user, as voiced recommendations ("Please tilt your wheelchair") or alarms ("Go back to a cooler location") to try to influence or correct behavior. Depending on the situation, they can be escalated as alarms to a caregiver or family member via email notification, SMS messages, or popup windows in a nursing station. Medical conditions such as abnormal HR or RR acquired from the BCG data are sent via email to the caregivers as notification in order to avoid unnecessary stress for the patient.

The data collected at the Boston Home show that lack of tilting, adverse ambient conditions, and apnea events do occur; hence, this system would be a useful addition to support daily activities in long-term wheelchair users. In this way, it is expected that the care of these patients will be improved without any discomfort, thus increasing their quality of life.

\section{Competing Interests}

The authors declare that there are no competing interests regarding the publication of this paper.

\section{Acknowledgments}

Esteban J. Pino would like to thank project Fondecyt Iniciación 11130340 for the financial support. Pablo Aqueveque wants to acknowledge Grant Fondequip EQM150114. The authors also would like to thank Marva Serotkin and Don Fredette from the Boston Home for their continuous support and the former students Dannie Araya, Belén Cavallar, and Javier Chávez for their work on the project.

\section{References}

[1] World Health Organization, Guidelines on the Provision of Manual Wheelchairs in Less Resourced Settings, World Health Organization, Geneva, Switzerland, 2008.

[2] The CNN Wire Staff, "Possible heat-related deaths reported in 3 states," The Blade, 2011.

[3] A. F. Henke, S. D. Cohle, and S. L. Cottingham, "Fatal hyperthermia secondary to sunbathing in a patient with multiple sclerosis," American Journal of Forensic Medicine and Pathology, vol. 21, no. 3, pp. 204-206, 2000.

[4] R. E. Kohlmeier, V. J. M. DiMaio, and K. Kagan-Hallet, "Fatal hyperthermia in hot baths in individuals with multiple sclerosis," American Journal of Forensic Medicine and Pathology, vol. 21, no. 3, pp. 201-203, 2000.

[5] F. K. Mutluay, H. N. Gürses, and S. Saip, "Effects of multiple sclerosis on respiratory functions," Clinical Rehabilitation, vol. 19, no. 4, pp. 426-432, 2005.

[6] J. Myers, M. Lee, and J. Kiratli, "Cardiovascular disease in spinal cord injury: an overview of prevalence, risk, evaluation, and management," American Journal of Physical Medicine \& Rehabilitation, vol. 86, no. 2, pp. 142-152, 2007.

[7] B. S. Kozniewski and J. L. Cezeaux, "Wheelchair pressure monitoring alert system for the reduction of the occurrence of pressure sores," in Proceedings of the IEEE 37th Annual Northeast Bioengineering Conference (NEBEC '11), pp. 1-2, IEEE, Troy, NY, USA, April 2011.

[8] J. G. Rocha, H. Carvalho, F. M. Duarte, M. A. F. Carvalho, and V. M. Moreira, "System providing discomfort monitoring for people in wheelchairs," in Proceedings of the IEEE International Symposium on Industrial Electronics, pp. 961-966, IEEE, Cambridge, Mass, USA, July 2008.

[9] R. Dai, S. E. Sonenblum, and S. Sprigle, "A robust wheelchair pressure relief monitoring system," in Proceedings of the 34th Annual International Conference of the IEEE Engineering in Medicine and Biology Society (EMBS '12), pp. 6107-6110, San Diego, Calif, USA, August 2012.

[10] Y.-S. Yang, G.-L. Chang, M.-J. Hsu, and J.-J. Chang, "Remote monitoring of sitting behaviors for community-dwelling manual wheelchair users with spinal cord injury," Spinal Cord, vol. 47, no. 1, pp. 67-71, 2009.

[11] R. P. Rush, "Sensation augmentation to relieve pressure sore formation in wheelchair users," in Proceedings of the 11th International ACM SIGACCESS Conference on Computers and Accessibility (Assets '09), pp. 275-276, ACM, October 2009.

[12] B. E. Dicianno, J. Arva, J. M. Lieberman et al., "RESNA position on the application of tilt, recline, and elevating legrests for wheelchairs," Assistive Technology: The Official Journal of RESNA, vol. 21, no. 1, pp. 13-22, 2009.

[13] D. Ding and E. Leister, "Usage of tilt-in-space, recline, and elevation seating functions in natural environment of wheelchair users," Journal of Rehabilitation Research \& Development, vol. 45, no. 7, pp. 973-983, 2008.

[14] D. Ding, R. A. Cooper, R. Cooper, and A. Kelleher, "Monitoring seat feature usage among wheelchair users," in Proceedings of the 29th Annual International Conference of the IEEE Engineering in Medicine and Biology Society (EMBC'07), pp. 4364-4367, IEEE, Lyon, France, August 2007.

[15] S. E. Sonenblum, S. Sprigle, and C. L. Maurer, "Use of power tilt systems in everyday life," Disability and Rehabilitation: Assistive Technology, vol. 4, no. 1, pp. 24-30, 2009.

[16] S. E. Sonenblum and S. Sprigle, "Distinct tilting behaviours with power tilt-in-space systems," Disability and Rehabilitation: Assistive Technology, vol. 6, no. 6, pp. 526-535, 2011.

[17] T. D. Yang, S. A. Hutchinson, L. A. Rice, K. L. Watkin, and Y.-K. Jan, "Development of a scalable monitoring system for wheelchair tilt-in-space usage," International Journal of Physical Medicine \& Rehabilitation, vol. 1, no. 4, article 129, 2013.

[18] D.-K. Han, J.-M. Kim, E.-J. Cha, and T.-S. Lee, "Wheelchair type biomedical system with event-recorder function," in Proceedings of the 30th Annual International Conference of the IEEE 
Engineering in Medicine and Biology Society (EMBS '08), pp. 1435-1438, IEEE, August 2008.

[19] O. A. Postolache, P. M. B. S. Girao, J. Mendes, E. C. Pinheiro, and G. Postolache, "Physiological parameters measurement based on wheelchair embedded sensors and advanced signal processing," IEEE Transactions on Instrumentation and Measurement, vol. 59, no. 10, pp. 2564-2574, 2010.

[20] P. Pancardo, J. A. Pardo, and J. C. Dueñas, "Automatic monitoring for workers healthcare in tropical climates," in Proceedings of the International Conference on Advanced Information Networking and Applications Workshops (WAINA '09), pp. 793-797, Bradford, UK, May 2009.

[21] D. Gun Park, S. Chul Shin, S. Won Kang, and Y. Tae Kim, "Development of flexible self adhesive patch for professional heat stress monitoring service," in Proceedings of the Annual International Conference of the IEEE Engineering in Medicine and Biology Society, vol. 4, pp. 3789-3792, 2005.

[22] E. J. Pino, D. E. Arias, P. Aqueveque, P. Melin, and D. W. Curtis, "Wireless pilot monitoring system for extreme race conditions," in Proceedings of the Annual International Conference of the IEEE Engineering in Medicine and Biology Society, pp. 5014-5017, San Diego, Calif, USA, August-September 2012.

[23] E. Pino, D. Arias, P. Aqueveque, and D. Curtis, "Assistive devices for healthcare: multiple sclerosis," in Proceedings of the AMIA Annual Symposium, p. 1899, Chicago, Ill, USA, November 2012.

[24] E. J. Pino, D. E. Arias, P. Aqueveque, L. Vilugron, D. Hermosilla, and D. W. Curtis, "Monitoring technology for wheelchair users with advanced multiple sclerosis," in Proceedings of the 35th Annual International Conference of the IEEE Engineering in Medicine and Biology Society (EMBC '13), pp. 961-964, Osaka, Japan, July 2013.

[25] D. E. Arias, E. J. Pino, P. Aqueveque, and D. W. Curtis, "Data collection capabilities of a new non-invasive monitoring system for patients with advanced multiple sclerosis," in Proceedings of the Annual Symposium Proceedings (AMIA '13), pp. 61-68, 2013.

[26] D. E. Arias, E. J. Pino, P. Aqueveque, and D. W. Curtis, "Daily activity monitoring for prevention of pressure ulcers in longterm wheelchair users," in Ambient Intelligence for Health, pp. 47-58, Springer International, Berlin, Germany, 2015.

[27] D. E. Arias, E. J. Pino, P. Aqueveque, and D. W. Curtis, "Wireless monitoring system for wheelchair users with severe mobility impairment," in Mobile Health: A Technology Road Map, vol. 5 of Springer Series in Bio-/Neuroinformatics, pp. 195-219, Springer, Berlin, Germany, 2015.

[28] The PostgreSQL Global Development Group, "PostgreSQL database," http://www.postgresql.org.

[29] M. J. Coggrave and L. S. Rose, "A specialist seating assessment clinic: changing pressure relief practice," Spinal Cord, vol. 41, no. 12, pp. 692-695, 2003.

[30] L. Chen, T. McKenna, A. Reisner, and J. Reifman, "Algorithms to qualify respiratory data collected during the transport of trauma patients," Physiological Measurement, vol. 27, no. 9, pp. 797-816, 2006.

[31] W. Lu, M. M. Nystrom, P. J. Parikh et al., "A semi-automatic method for peak and valley detection in free-breathing respiratory waveforms," Medical Physics, vol. 33, no. 10, pp. 3634-3636, 2006.

[32] R. Johnson, "The influence of dew-point temperature on multiple sclerosis symptoms," Weather, vol. 60, no. 6, pp. 170-170, 2005.
[33] J. F. Kurtzke, "Rating neurologic impairment in multiple sclerosis: an expanded disability status scale (EDSS)," Neurology, vol. 33, no. 11, pp. 1444-1452, 1983.

[34] Consortium for Spinal Cord Medicine, Pressure Ulcer Prevention and Treatment Following Spinal Cord Injury: A Clinical Practice Guideline for Health-Care Professionals, Paralyzed Veterans of America, 2000.

[35] R. S. Porter, J. L. Kaplan, and B. P. Homeier, The Merck Manual Home Health Handbook, Merck, Rahway, NJ, USA, 2009.

[36] T. Kasai and T. D. Bradley, "Obstructive sleep apnea and heart failure: pathophysiologic and therapeutic implications," Journal of the American College of Cardiology, vol. 57, no. 2, pp. 119-127, 2011.

[37] G. Fortino, R. Giannantonio, R. Gravina, P. Kuryloski, and R. Jafari, "Enabling effective programming and flexible management of efficient body sensor network applications," IEEE Transactions on Human-Machine Systems, vol. 43, no. 1, pp. 115133, 2013.

[38] G. Fortino, S. Galzarano, R. Gravina, and W. Li, "A framework for collaborative computing and multi-sensor data fusion in body sensor networks," Information Fusion, vol. 22, pp. 50-70, 2015.

[39] V. Janardhan and R. Bakshi, "Quality of life in patients with multiple sclerosis: the impact of fatigue and depression," Journal of the Neurological Sciences, vol. 205, no. 1, pp. 51-58, 2002. 

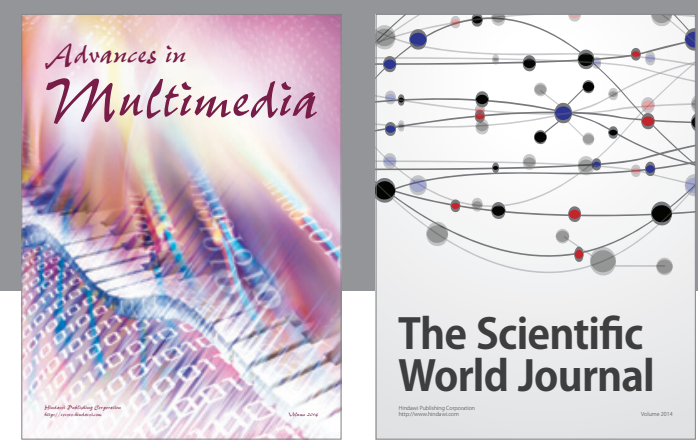

The Scientific World Journal
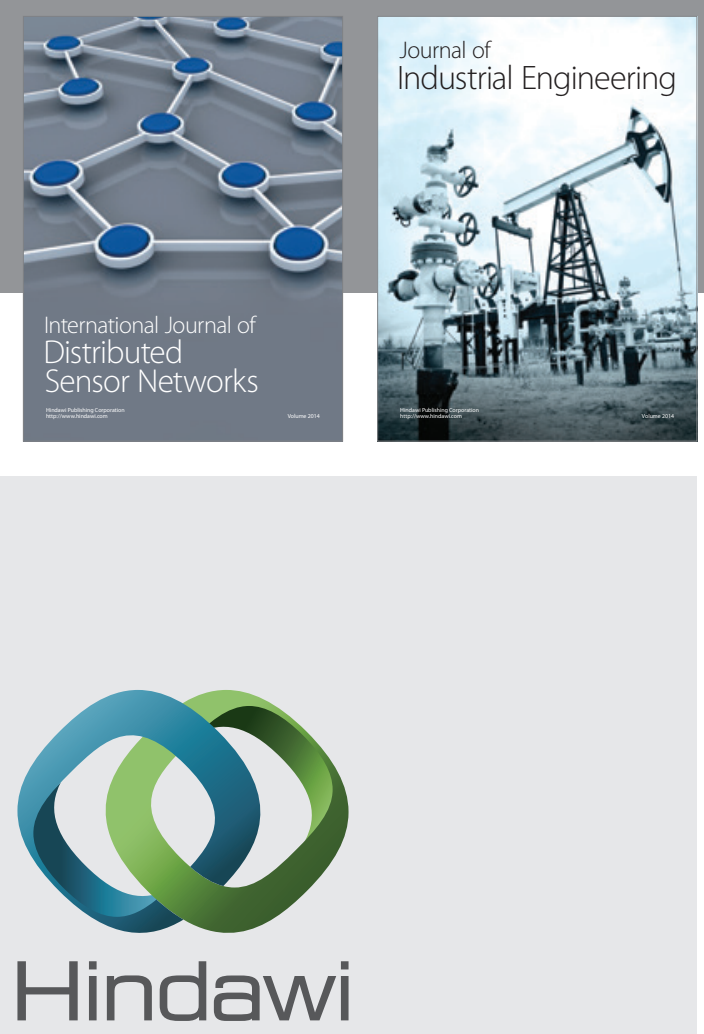

Submit your manuscripts at

http://www.hindawi.com

\section{Computer Networks} and Communications
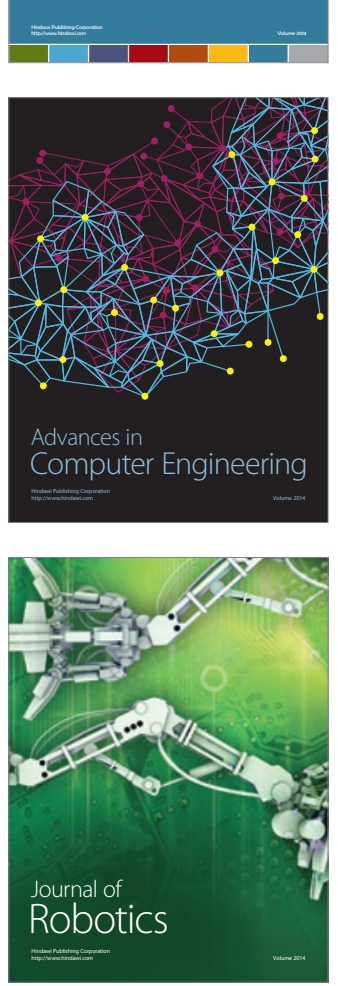
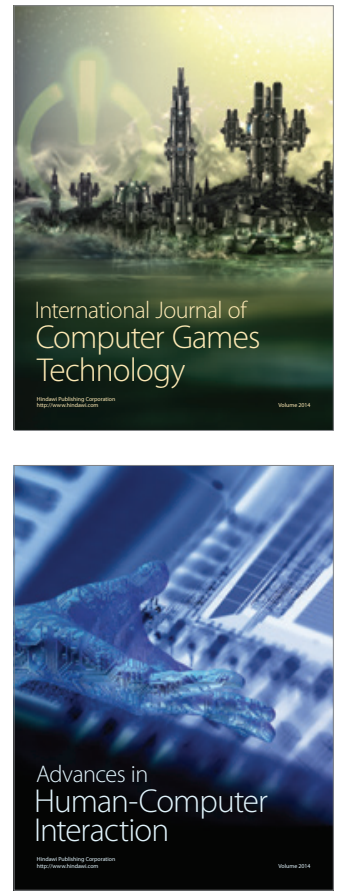
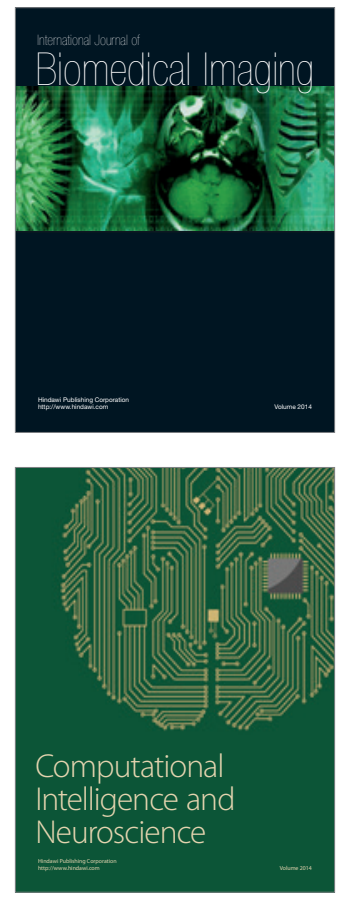
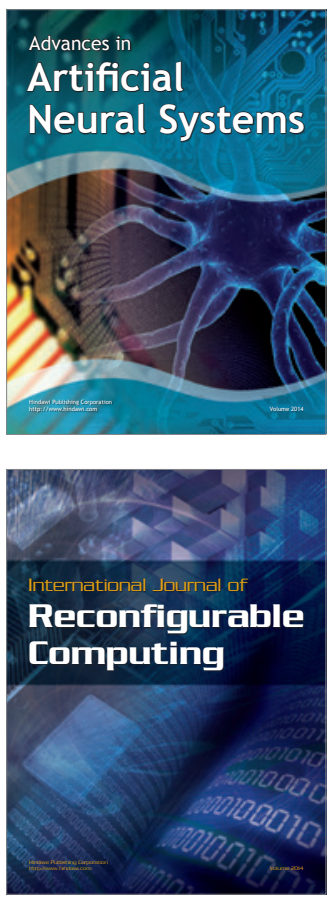
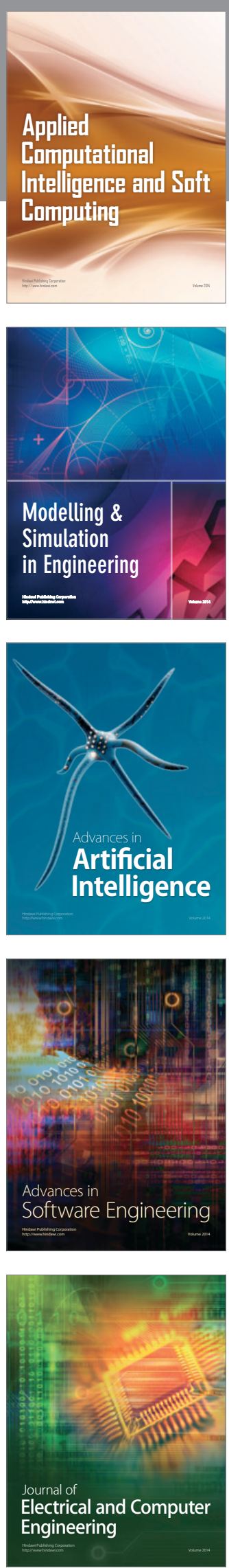